\title{
Data Mining: An Effective Tool in Cognitive Psychological Emotion Analysis \\ SUNitA VERMA ${ }^{1}$, Dr. ANAMika AHIRWAR ${ }^{2}$ \\ Research Scholar ${ }^{1}$, Associate Professor ${ }^{2}$ \\ Dept. of Science \& Technology \\ Jayoti Vidyapeeth Women's University \\ Jaipur (Raj.) \\ Email-dranamikaahirwar@jvwu.ac.in
}

\begin{abstract}
Data Mining is an aggregate of various techniques and methods to extract out information from a large database in simple, understandable and usable form. A variety of data mining techniques has been applied successfully in the health care system and is attracting the attention of scientist and researchers throughout the world. The present research study describes various cognitive psychological theories of emotions and applications of different data mining methods. The study also emphasizes the data mining methods significant in the cognitive psychological emotion analysis.
\end{abstract}

Keywords- Data mining, cognitive, emotion, clustering, decision tree

\section{Introduction}

Data mining is an effective instrument which recognizes patterns and relationships in the data and extracts useful information from a huge set of data base. The ultimate objective of this process is to extract information from a data set 
and convert it into an understandable form for easy usage. Data mining methods have shaped as a branch of applied artificial intelligence. Significant development in computer technology has led to the introduction of new, better and improved technologies for web-based education. The tremendous growth in databases has fostered the need to develop technologies that can utilize information and knowledge efficiently, thereby responsible for the increasing demand of data mining techniques. Some significant types of data mining methods include generalization, characterization, classification, clustering, association, evolution, pattern matching, data visualization and meta-rule guided mining etc.

The objective of the present research paper is to highlight various data mining methods which can be useful in cognitive psychological emotion analysis.

\section{Cognitive Theory of Emotion}

Emotions are an integral part of human life and when represented in pathological terms such as psychiatric disorders of emotional regulation and control, (depressive and bipolar disorders), they are primary reason for medical disability. Although the significance of emotion in human health and illness is well established, scientist and researchers have varied opinions on emotional phenomena, experiences and analysis (LeDoux, 1995; LeDoux, 2012).

Cognitive psychology may be defined as a branch of medical science which deals with the study of internal mental processes such as perception, thinking, memory, attention, and language, problem solving and learning. Cognitive psychologists try to build up mental models of the data processing for these processes occurring inside brain. The advancement in the field of computer and technology has ushered the cognitive psychological research of human brain. 
It has helped psychologists to understand the complexities of human cognition in a better efficient way.

The "Cognitive Theory of Emotion" signifies a family of feeling philosophies, urbanized typically in psychology and philosophy, which share the guess that feelings (the periodic states of individuals indicated in usual verbal by arguments like "enjoyment," "sorrow," "expectation," "panic," etc.), or at most a core subsection of feelings, presume for their presence assured perceptions almost the producing events. Usually it is expected that these emotion-relevant cognitions determine, alone or together with non-cognitive mental states (in particular, desires), whether or not an event elicits an emotion, the kind of feeling it produces (e. g., joy, sadness) and the intensity of the elicited emotion (e.g., weak enjoyment, intense joy). The theme of emotion philosophies is huge and miscellaneous. Tremendous work has been done on the "Basic emotion theory" thereby helping psychopathologists in this field (Williams, 2017; Wang et al., 2018). Basic emotion theory states that humans have only a few significant emotions such as fear, anger, joy and sadness which can be considered basic in biological and psychological terms (Wilson-Mendenhall et al., 2013). Various theories regarding emotions have been given from time to time such as Ekman's Theory of Basic Emotions, Plutchik's Wheel of Emotions, Russel's Circumplex Model etc.

\section{Data mining and its application}

Data mining is a pool of techniques for competent automated finding of previously unknown, valid, novel, useful and understandable patterns in large 
databases. A few significant data mining techniques and their applications are mentioned below (Liao et al., 2012):-

- Neural networks and their applications- The term, neural network, is traditionally assigned to a system of biological neurons. In modern terminology the term refers to artificial neural networks composed of artificial neurons or nodes, electrical signaling and other forms of signaling which ascend from neural transmitter diffusion effecting electrical signaling. These are very complex and are involved in very complicated applications such as radial basis function networks, neural classification, Bayesian confidence propagation neural networks, gene regulatory networks, fuzzy recurrent neural networks, neural nets, back-propagation artificial neural networks, general regression neural networks and flow networks.

- Algorithm architecture and its applications - Algorithm architecture is a finite list of well-defined instructions for function calculations. Algorithms are utilized in calculation, data processing and automated reasoning. In simple terms "an algorithm is a step-by-step process for calculation." Algorithms find application in gap statistic algorithms, chi-square automated interaction detection, models and algorithms, GRASP, OLAP, k-means, Clustering algorithms, decision forest algorithms, classification and regression trees, Euclidean distance, bagged clustering algorithms, fuzzy logic, association rules, C\&RT, Apriori algorithms, C5, anomaly-based IDS, clustering, genetic algorithms, CRISP-DM models, thyroid stimulation and SVM. 
- Dynamic prediction based approach and its applications - This is a mathematical model for stochastic dynamics. Some applications which use a dynamic prediction based approach include ophthalmic oncology, vehicle fault diagnosis, grid computing, dyadic wavelet, pre-fetching, fault restoration prediction models, fault prediction models, financial distress prediction models, Vlasov-Maxwell equations, chemical reactivity predictions, real time vehicle tracking, forecasting, anomaly detection, churn prediction, comparative genomics, clinical predictions and predictive models.

- Analysis of system architecture and its applications - The analysis of system architecture uses a conceptual model that defines the structure, behavior and other aspects of a system. It utilizes both hardware and software for designing composite systems. The significant applications include semantic analysis, regression analysis, statistical analysis, discriminative analysis, association analysis, penalized discriminative analysis, process parameter analysis, cluster analysis, decision making, decision support systems, consumer behavior analysis, binary logistical regression analyses, M5 model trees, factor analysis, market basket analysis, collaborative filtering, data analysis, decision tree based models, principal component analysis, multifeature selection, intrusion detection and hem dialysis.

- Intelligence agent systems and their applications - In the field of artificial intelligence, an intelligent agent system is an autonomous entity which is based on acting upon observation. Intelligent agents are involved in learning and utilizing the gained knowledge to fulfill desired objectives. Some 
applications for intelligence agent systems include multi-agent systems, complex systems, computer interface design, multiuser database systems, intelligent analysis, manufacturing intelligence, intelligent tutoring systems, support vector machines, program diagnostics systems, supervisory and specialist systems, supervisory and specialist systems, computing intelligence, artificial intelligence and Mahalanobis Taguchi systems.

- Modeling and its applications - Modeling technology involves quantitative methods for the data analysis, acquire and represents expert knowledge, using inductive logic programming, algorithms, so that AI, cognitive science and other research fields are benefited for the development of data mining technology. Applications of modeling include cost modeling, model-based diagnosis, forest fire proliferation modeling, model output statistics, intonation modeling, XML document modeling, Cox proportional hazard modeling, load damage exponents, polynomials, similar waveforms, simple additive weight, computer numerical control, meta learning and drug utilization.

- Knowledge-based systems and their applications - These are narrow domain working tools which give better efficient decisions. Knowledge in these systems is attained and characterized, using various knowledge illustration techniques, rules, frames and scripts. Some applications of knowledge-based systems include learning techniques, auto control techniques, and knowledge discovery in databases, knowledge spirals, communication technologies, knowledge measurement, knowledge extraction, knowledge acquisition, 
knowledge management, knowledge representation, digital libraries and information gain theory data mining.

- System optimization and its applications - System Optimization denotes the selection of a best element, from some set of available alternatives. Important applications of system optimization are- electrical nerve stimulation, R-peak detection, latent reference individual extraction methods, operation optimization values, vertical partitioning, logistical regression, analytical hierarchy processes, polynomial regression, biogeography based optimization, particle swarm optimization, finite element methods, discrete rough set methods, asymptotic methods and parallel computing.

- Information systems and their applications - Information systems are the harvests of an academic discipline. They are a bridge between the business and computer domain. An information system is based on the theoretical fundamentals of information and computing which permit researchers to engage in academic studies of various business models and related algorithmic processes that are relevant to computer science. In general, information systems concentrate on the processing of information in any organizations, especially within business enterprises. These products can be beneficial for the society as well. The applications of information systems include patient characteristics, catchment characteristics, mobile databases, self-organizing, feature maps, insurance claims databases, alternating current field measurement, fracture acidizing, latest time sub-series, destination choice, attribute relevance studies, fraudulent financial statements, sequence 
similarity, case-based reasoning, anthropometric data, regression spines, economic imbalances, medium-voltage customer faults, maintenance and engineering, bank lending, reinforcement learning, supervised learning, arousals, information visualization, customer retention, churn management, pattern discovery, customer relationship management and uniaxial compressive strength.

\section{Data mining and Cognitive Psychological Emotion Analysis}

Emotions are an integral part of human perception along with their memory, thinking and imagination. Human perception occupies a significant place present times including business, education, art, literature, music and everyday life of every individual. Thus emotions also are an inseparable part of today's world. Many important methods of Data mining find significant application in human emotions research.

Clustering is one such important method which is at the center of data analysis and data mining applications. The capacity to find highly correlated regions of objects when their number becomes very large, is highly desirable, as data sets grow and their characteristics and data interrelationships vary. Thus clustering may be defined as the separation of the objects into specific groups based on certain characteristics. Our brain is a storehouse of various types of emotions and a detailed analysis of these emotions can lead us to the cause behind the variations in these emotions. For achieving this, classification is done which is a process of selecting a model that defines and differentiates various data classes. Before proceeding towards classification, the method of clustering is required. 
Various clustering techniques are used for human emotion studies (Jaya and Kumar, 2016).

- $\quad$ K-Means Clustering: It is an efficient, less complicated and simple method. It aims to group observations into $\mathrm{K}$ clusters in which observations belong to the cluster with the nearest mean serving as the prototype of the cluster. The results may vary in the presence of outlier.

- Hierarchical Clustering: It is implementation is easier and the visualization capability is good. No necessity of mentioning the clusters in advance. It is slower as it has cubic time complexity in many cases. Once a decision is made it cannot be withdrawn. It will not work proper in the presence of noise. It is not scalable one.

- Density based Clustering: It refers to unsupervised learning methods that identify distinctive clusters in the data, based on the idea that a cluster in a data space is a contiguous region of high point density, separated from other such clusters by contiguous regions of low point density. In this case there is no requirement for the specification of number of clusters in advance. It will work well in the presence of noise. It will not handle the data points with varying densities. Results are based on the distance measured.

Another important method is the Decision Tree. Decision Tree induction encompasses learning and classification. DT is similar to the flowchart in which every non-leaf nodes denotes a test on a particular attribute and every branch denotes an outcome of that test and every leaf node have a class label. The node at the top most labels in the tree is called root node. The steps are simple and can be applied to any domain. It is advantageous over other methods as decision 
trees can be visualized and are very simple to understand and interpret. They require least data preparation in comparison to other techniques. It can handle both categorical and numerical data whereas other techniques are for a single type of variable. It can also deal in multi-output problems (Sharma and Kumar, 2016).

Various other techniques such as support vector machine (svm), Neural network, Bayesian method, Association, Regression etc are commonly used for data mining in health care including emotion analysis.

\section{Conclusion}

Thus to conclude, it can be stated beyond doubt that the data mining techniques such as clustering, decision tree and many more can be very helpful in extracting useful inferential information from a huge set of data in the field of health care including the cognitive psychological studies.

\section{References}

1. Jaya, R., \& Kumar, S. M. (2016). A Study on Data Mining Techniques Methods Tools and Applications in Various Industries. International Journal of Current Research and Review, 8 (4), 34.

2. LeDoux, J. E. (1995). Emotion: Clues from the brain. Annual review of psychology, 46 (1), 209-235.

3. LeDoux, J. E. (2012). Evolution of human emotion: a view through fear. Progress in brain research, 195, 431-442. 
4. Liao, S. H., Chu, P. H., \& Hsiao, P. Y. (2012). Data mining techniques and applications-A decade review from 2000 to 2011. Expert systems with applications, 39 (12), 11303-11311.

5. Sharma, H., \& Kumar, S. (2016). A survey on decision tree algorithms of classification in data mining. International Journal of Science and Research (IJSR), 5(4), 2094-2097.

6. Wang, F., Pan, F., Shapiro, L. A., and Huang, J. H. (2018). Stress induced neuroplasticity and mental disorders 2018. Neural Plast. 2018:5382537.

7. Williams, R. (2017). Anger as a basic emotion and its role in personality building and pathological growth: the neuroscientific, developmental and clinical perspectives. Front. Psychol. 8:1950.

8. Wilson-Mendenhall, C. D., Barrett, L. F., \& Barsalou, L. W. (2013). Neural evidence that human emotions share core affective properties. Psychological science, 24 (6), 947-956. 